\title{
Morphometric study of Scapular glenoid cavity in Indian population
}

\author{
Dr. Gosavi S.N. ${ }^{1}$, Dr. Jadhav S. D. ${ }^{2}$, Dr. Garud R.S. ${ }^{3}$ \\ ${ }^{1,3}$ Dept. of Anatomy, BVDU Medical College, Pune/ Bharati Vidyapeeth/India \\ ${ }^{2}$ Dept. of Anatomy, PDVVPF's Medical College, Ahmednagar/MUHS/ India
}

\begin{abstract}
Knowledge of the shape and dimensions of the glenoid cavity are important in the design and proper fitting of glenoid component for total shoulder arthroplasty. One hundred and forty two dry scapulae (62 right and 80 left) of unknown sex and age, belonging to Indian population, were examined using digital vernier caliper. The height and anteroposterior width of the glenoid cavity was measured at three different levels. Shape of the glenoid cavity, based on the presence of a notch on the anterior glenoid rim, was noted as pear, oval or inverted comma shape. The mean height of the glenoid cavity was observed as $35.03 \pm 5.25 \mathrm{~mm}$ on the right side and $35.3 \pm 3.41 \mathrm{~mm}$ on the left side. The Anteroposterior diameter was $24.17 \pm 2.57 \mathrm{~mm}$ on the right and $23.9 \pm$ $2.66 \mathrm{~mm}$ on the left side. $49.9 \%$ glenoid cavities were pear shape, $38 \%$ were oval and $12.05 \%$ were of inverted comma shape. Size of the glenoid cavity is variable. Hence while designing the glenoid component of the prosthesis; the smaller dimensions of the glenoid cavities in Indian population should be considered.
\end{abstract}

Key words: Glenoid cavity, Shoulder prosthesis, Morphometry

\section{Introduction}

Knowledge of the shape and dimensions of the glenoid are important in the design and fitting of glenoid component for total shoulder arthroplasty. An understanding of variations in normal anatomy of glenoid is essential while evaluating pathological conditions like osseous Bankart lesion and osteochondral defects. [1]

Anatomical parameters of the glenoid relevant to prosthesis design include glenoid height, width, articular surface area, inclination, vault size and shape. A number of cadaveric studies have demonstrated considerable natural variability in these parameters; this variability affects prosthesis design, instrumentation and intraoperative implantation techniques. [2]

The shape of the glenoid cavity has been diversely described as teardrop or pear shaped, round, ovoid and an inverted comma-shaped depending on the presence or absence of a notch on the anterior glenoid rim.[1] According to Fick et al. [3] the glenoid notch is situated somewhat above the middle of the anterior margin of the cavity and can be very prominent, very shallow or absent. When the notch is present the shape of the cavity can be described as pear shaped or inverted comma shaped. When it is absent the cavity is round or oval shaped. [4]

With this background in mind, we studied the glenoid cavities of scapulae, belonging to Indian population, to gather the morphometrical data, which can be useful for designing of glenoid component of shoulder prosthesis.

\section{Materials and Methods}

We studied 142 dry scapulae (62 right and 80 left) belonging to Indian population of unknown sex and age. Broken, damaged or scapulae showing degenerative changes were excluded from the study. Following parameters were studied using digital vernier caliper.

(i)Height of glenoid cavity (Ht) was measured as maximum distance between the most prominent point on the supra-glenoid tubercle to the inferior margin of the glenoid cavity. (Fig.1)

(ii) The width of the glenoid was measured at three different levels. Anteroposterior width (AP1) was measured as the maximum breadth perpendicular to the height of glenoid in the lower half. Anteroposterior width (AP2) was measured as AP diameter of upper half of the glenoid cavity midway between superior margin and midequater. The diameter at the level of the peak of the glenoid notch, when present, was also measured (AP3).

(iii) Shape of the glenoid cavity was noted as oval, pear shaped or inverted comma shape. All the observations were done by a single observer.

The mean, standard deviation, range and asymmetry in bilateral parameters using t-test was calculated using SPSS 17 software for the data. $P$ values $<0.05$ were considered significant.

\section{Results}

We observed 142 dry scapulae of unknown sex. (Table 1\&2). The mean height of the glenoid cavity was observed as $35.03 \pm 5.25 \mathrm{~mm}$ on the right side and $35.3 \pm 3.41 \mathrm{~mm}$ on the left side. The Anteroposterior 
diameter (AP1) was $24.17 \pm 2.57 \mathrm{~mm}$ on the right and $23.9 \pm 2.66 \mathrm{~mm}$ on the left side. The average diameter measured in the upper half of the glenoid cavity (AP2) was $14.58 \mathrm{~mm}$. While AP3, measured at the level of glenoid notch present on the anterior glenoid rim, on the right side $(\mathrm{n}=42)$ was $17.72 \pm 1.89 \mathrm{~mm}$ and on the left side $(\mathrm{n}=45)$ was $16.82 \pm 2.18 \mathrm{~mm}$. The bilateral difference in AP3 was statistically significant. $(\mathrm{p}<0.05)$

Majority of glenoid cavities were pear shaped $(54.83 \%$ on the right side and $45 \%$ on the left side). Oval shape was found in $32.25 \%$ glenoids on the right side and $43.75 \%$ of left the side. Inverted comma shape was observed in $12.9 \%$ of the right sided and in $11.2 \%$ of the left sided cavities.

\section{Discussion}

The morphology of the glenoid cavity is highly variable and no consensus exits regarding how to classify the different forms. Nevertheless, there is an agreement on the function of the glenoid cavity: an osseous base is provided for the stability of the glenohumoral joint both sagittally and vertically.[5]

The shoulder joint is the most frequently dislocated joint in the body. Dislocations with fractures of the glenoid are also quite common in trauma. [1] For management of such injuries, prosthesis and arthroplasty are frequently required. The anatomical basis and variations of shape and size of glenoid cavity of scapula is of fundamental importance in understanding of rotator cuff disease, shoulder dislocation and to decide the proper size of the glenoid component in the shoulder arhtroplasty.[6]

The mean height of the glenoid cavity in the present study was $35.16 \mathrm{~mm}$, Mamatha et al. [1] observed it as $33.79 \mathrm{~mm}$, Rajput et al.[6] as $34.59 \mathrm{~mm}$. and Dhindsa et al [7] as $34.24 \mathrm{~mm}$. Middernacht et al.[8] noted it as $37.3 \mathrm{~mm}$ and Coskun et al [9] as $36.3 \mathrm{~mm}$.(Table 3)

The mean AP1 diameter measured as the maximum diameter in the lower half of glenoid cavity was 24 $\mathrm{mm}$ in the present study which is similar to the observations by Mamatha et al. [1] (23.2 mm), Rajput et al. [6] $(23.11 \mathrm{~mm})$ and Dhindsa et al [7] $(23.70 \mathrm{~mm})$. Middernacht et al.[8] observed it as $27.9 \mathrm{~mm}$ and Coskun et al [9] as $24.6 \mathrm{~mm}$.(Table 3)

Mean anteroposterior diameter (AP2) measured in the upper half of the glenoid cavity was $14.58 \mathrm{~mm}$ in the present study. Our findings were similar to that of Rajput et al. [6] (14.46 mm), but less than the observations by Mamatha et al. [1] (16.02 mm). (Table 3)

The mean Anteroposterior diameter measured at the peak of the glenoid notch (AP3) was $17.27 \mathrm{~mm}$, which was slightly greater than the observations by Rajput et al. [6] (15.72 $\mathrm{mm}$ ). (Table 3)

Various shapes of the glenoid cavity have been described based on the presence of a notch on the anterior glenoid rim. It has been found that if the notch is distinct, then the glenoid labrum is not fixed to the bony margin of the notch but bridges the notch itself. This could make the shoulder joint less resistant to dislocating forces.[4] Anetzberger and Putz,[10] classified the shape of the glenoid cavity as teardrop, type IA and IB, with and without a notch respectively, from an elongated oval shape type II.

In the present study we observed more number of pear shaped and oval but less number of inverted comma shaped glenoid cavities (Pear shaped $-49.91 \%$, Oval $-38 \%$, Inverted comma $-12.05 \%$ ), as compared to Mamatha et al. [1]( Pear shaped - 44.5\%, Oval -22\%, Inverted comma - 33.5\%) and Rajput et al. [6] (Pear shaped $-47.5 \%$, Oval $-15.5 \%$, Inverted comma -36\%). Prescher and Klumpen [4] observed oval shaped glenoids in $35 \%$ and pear shaped in $30 \%$. While Coskun et al [9] observed $28 \%$ glenoids as pear shaped and $72 \%$ were oval in shape.

We are in agreement with Mamatha et al [1] that, there was no priority of the side for the glenoid notch, so that an influence of handedness on the shaping of the glenoid cavity is unlikely.

Mathew et al.[11], observed that, in south India, $16.5 \%$ of type 2 diabetes mellitus patients suffered from frozen shoulder, $5.48 \%$ from periathritis of the shoulder joint and $3.55 \%$ from rotator cuff injury. An increase in the aging population accompanied by an increase in the incidence of type 2 diabetes mellitus indicates that arthritic disease of the shoulder may become more common in the near future. [1] It has also been observed that, the shoulder is the third most common large joint to require surgical reconstruction, after the knee and hip.[12]

In their morphometric study of glenoid cavities in Indian population, Mamatha et al.[1], Rajput et al. [6], and Dhindsa et al. [7], found that, all the parameters are smaller than the similar measurements in other population. Mamatha et al[1] observed that, the standard available smallest glenoid component $(40 \mathrm{~mm})$ may not fit the glenoid in their study population. The findings of the present study corroborates with their findings.

\section{Conclusion}

Size of the glenoid cavity is known to be variable in different population. Hence while designing the glenoid component of the prosthesis; the smaller dimensions of the glenoid cavities in Indian population should be considered. 


\section{References}

[1] Mamatha T, Pai SR, Murlimanju BV, Kalthur SG, Pai MM, Kumar B. Morphology of glenoid cavity. Online J health Allied Scs. 10(3), 2011, 7 .

[2] Strauss EJ, Roche C, Flurin PH, Wright T, Zuckerman JD. The glenoid in shoulder arthroplasty. J shoulder Elbow Surg, 18, 2009, 819-833.

[3] Fick R Anatomie der Gelenke. Handbuch der Anatomie des Menschen. (ed. Von Bardelben K) 1904 Bd II, Abt 1, TI 1. Jena: G Fischer.

[4] Prescher A, Klumpen T. The glenoid notch and its relation to the shape of the glenoid cavity. J. Anat 190, 1997, 457-460.

[5] Wild LFDe, Berghs BM, Audenaert E, Sys G, Van Maele GO, Barbaix E. About the variability of the shape of the glenoid cavity. Surg Radiol Anat 26, 2004, 54-59.

[6] Rajput HB, Vyas KK, Shroff BD. A study of morphological patterns of the glenoid cavity of scapula National J of Medical Research 2(4, 2012, 504-507.

[7] Dhindsa GS, Singh Z. A study of morphology of glenoid cavity. Jr of Evolution of Medical and Dental sciences, 3, 2014, 70367043.

[8] Middernacht B, Pieter-Jan De Roo, Maele GV, Wild LFDe. Consequences of scapular anatomy for Reversed total shoulder arhtroplasty. Clin Orthop Relat Res, 466, 2008, 1410 - 1418.

[9] Coskun N, Karaali K, Cevikol C, Demirel BM, Sindel M. Anatomical basics and variations of the scapula in Turkish adults. Saudi Med J 27(9), 2006, 1320-1325.

[10] Anetzberger H, Putz R. The scapula: principles of construction and stress. Acta Anat Basel 156, 1996, 70-80

[11] Mathew AJ, Nair JB, Pillai SS. Rheumatic musculoskeletal manifestations in type 2 diabetes mellitus in south India. Int J Rheum Dis 14, 2011, 55-60.

[12] Kabak LA, Green A, Blaine TA. Glenohumoral arthritis and total shoulder replacement. Medicine \& Health/ Rhodes Island. 95(4 , 2012, 120-124.

Table I: Observations of various parameters of the glenoid cavity. (All values in $\mathrm{mm}$ )

\begin{tabular}{llllll}
\hline Parameter & Right & Left & Mean & Range & T value \\
\hline Ht. & $35.03 \pm 5.25$ & $35.3 \pm 3.41$ & 35.16 & $28.1-47.4$ & 0.35 \\
AP1 & $24.17 \pm 2.57$ & $23.9 \pm 2.66$ & 24 & $13.8-30.2$ & 0.61 \\
AP2 & $14.56 \pm 2.03$ & $14.6 \pm 1.85$ & 14.58 & $10.9-20$ & $>0.05$ \\
AP3 & $17.72 \pm 1.89(\mathrm{n}=42)$ & $16.82 \pm 2.18$ & 17.27 & $13-22$ & $>0.05$ \\
& & $(\mathrm{n}=45)$ & & & 2.08 \\
\hline
\end{tabular}

Table legends: Ht. - vertical height of the glenoid cavity, AP - antero-posterior diameter

Table II: Table showing the shapes of glenoid cavity

\begin{tabular}{lll}
\hline Shape & Right $(\mathbf{n}=\mathbf{6 2})$ & Left $(\mathbf{n}=\mathbf{8 0})$ \\
\hline Oval & $20(32.25 \%)$ & $35(43.75 \%)$ \\
Pear & $34(54.83 \%)$ & $36(45 \%)$ \\
Inverted comma & $08(12.9 \%)$ & $09(11.2 \%)$ \\
\hline
\end{tabular}

Table III: Comparison of parameters of glenoid in various studies

\begin{tabular}{|c|c|c|c|c|c|}
\hline & $\begin{array}{l}\text { Population } \\
\text { studied }\end{array}$ & Ht. & AP1 & AP2 & AP3 \\
\hline Mamatha et al [1] & India & 33.79 & 23.2 & 16.02 & \\
\hline Rajput et al et al[6[ & India & 34.59 & 23.11 & 14.46 & 15.72 \\
\hline Dhindsa et al[7] & India & 34.24 & 23.70 & & \\
\hline Middernsche et al[8] & Belgium & 37.3 & 27.9 & & \\
\hline Coskun et al[9] & Turkey & 36.3 & 24.6 & & \\
\hline Present study & India & 35.16 & 24.0 & 14.58 & 17.27 \\
\hline
\end{tabular}

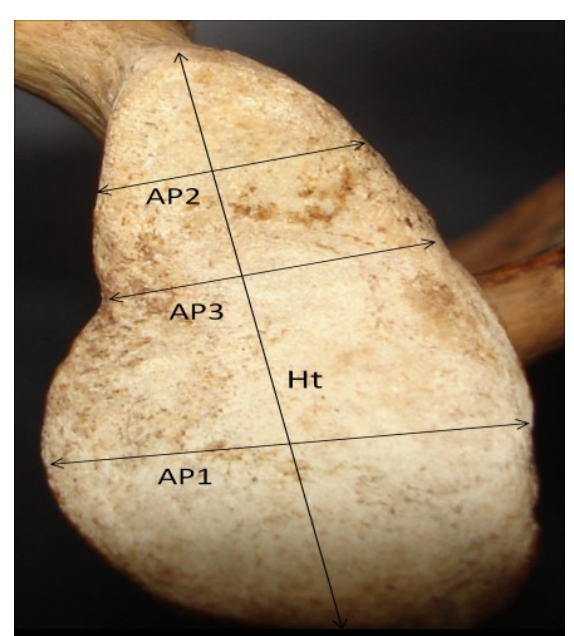

Figure 1: Diagram showing the various parameters

$\mathrm{Ht}$ - Height of the glenoid cavity, AP - Antero-posterior diameter 